\title{
Spatiotemporal analysis of the land surface temperature distribution over the territory of Novosibirsk city based on Landsat data
}

\author{
Elena A. Mamash ${ }^{1, *}$, Igor A. Pestunov ${ }^{1}$, and Dmitri L. Chubarov ${ }^{1}$ \\ ${ }^{1}$ Federal Research Center for Information and Computational Technologies, Novosibirsk, Russia
}

\begin{abstract}
The paper discusses the applicability of Landsat 8 data for analyzing the land surface temperature distribution over the territory of Novosibirsk. The satellite data is compared with the data from ground meteorological stations. Using cloud-based systems and methods for processing time series of satellite data, a composite image of the temperature field for the territory of Novosibirsk is built; trends and anomalies in the temperature distribution in the urban area are studied. The results could be applied in urban development analysis and management of the city territory.
\end{abstract}

Availability of remote sensing data of low, moderate and high spatial resolution gave rise to development of tools for its online processing. Probably the most widely used such tool is Google Earth Engine, other examples include VEGA-Science developed at the Space Research Institute of Russian Academy of Science [1]. Federal Research Center for Information and Computational Technologies maintains an archive of satellite remote sensing data accumulated since 2007 and an information system for online retrieval and processing of satellite data time series [2].

Transition from individual satellite images to time series makes it possible not only to follow the dynamics of change but to compute statistical characteristics of different environmental characteristics such as land surface temperature (LST) of a certain region. This in turn permits further statistical processing such as removal of outliers and locating persistent anomalies.

Land surface temperature retrieved from Landsat data is used extensively for many applications including observation of urban heat islands, locating persistent anomalies, and monitoring urban sprawl. Examples include studies of urban heat islands of cities located in moderate climate zones such as Moscow and Nizhny Novgorod, as well as largest cities located above the Polar Circle, the cities of Murmansk, Norilsk, Vorkuta, Apatity, Salekhard, Noviy Urengoy, Nadym [3-7]. Detection of changes of the urban landscape of Krasnoyarsk was performed using the land surface temperature data retrieved from thermal infrared satellite imagery [8]. We remark that the authors of the papers cited above use individual satellite scenes for mapping and use pairs of images for the task of change detection. We hypothesize that such methods might be sensitive to the choice of images.

The uncertainty in satellite measurements of land surface temperature can be quantified by comparing LST retrievals with the data from ground weather stations [9].

\footnotetext{
*e-mail: elenamamash@gmail.com
} 
To retrieve land surface temperature from Landsat data we apply the single channel algorithm $[10,11]$.

$$
T_{L S T}=\frac{T_{B}}{\left(1+\lambda \frac{T_{B}}{c_{2}}\right) \cdot \ln \varepsilon}-273.15,
$$

where $T_{B}$ denotes the brightness temperature $(\mathrm{K})$ determined from observed radiation intensity in a satellite spectral channel, $\lambda$ - the central wavelength of the satellite spectral channel, for Landsat Band 10 it equals $10.8 \mu \mathrm{m}, c_{2}=h \cdot c / k_{B}=1.4388 \times 10^{-2} \mathrm{~m} \mathrm{~K}=14388 \mu \mathrm{m} \mathrm{K}$ is the second Planck radiation constant expressed as the product of Planck constant $h=$ $6.626 \times 10^{-34} \mathrm{~J} \mathrm{~s} ; k_{B}=1.38 \times 10^{-23} \mathrm{~J} \mathrm{~K}^{-1}$ the Boltzmann constant; $c=2.998 \times 10^{8} \mathrm{~m} \mathrm{~s}^{-1}$ is the speed of light; and $\varepsilon$ is the surface emissivity.

Several approaches to estimating surface emissivity can be found in the literature. The most commonly used are (1) $\varepsilon$ is considered constant usually equal to 0.98 ; (2) land use classification is performed and each class is assigned a specific emissivity coefficient (3) the emissivity coefficient is estimated from NDVI [12]. In this study we exclusively use the emissivity coefficient estimated from NDVI.

We compared the temperature values obtained with the Equation 1 with emissivity estimated from NDVI and with emissivity set constant at 0.98 and encountered differences that did not exceed a tenth of a degree. Therefore we conclude that the temperature is not particularly sensitive to this parameter.

When computing temporal composites we applied masks to exclude clouds and cloud shadows extracted from Landsat QA band.

To perform the computation and compose the multitemporal images we used Google Earth Engine. This instrument provides access to Landsat 8 data processed with atmospheric correction ${ }^{1}$.

Landsat 8 thermal bands have spatial resolution of approximately 100 meters however in Google Earth Engine this data is resampled to 30 meters which makes this data particularly suitable for use at an urban scale.

We compare the land surface temperature maps with the land use data obtained from NextGIS ${ }^{2}$ and map data from Google Maps and Bing Maps.

To validate the temperature data retrieved from Landsat we used the measurements from the five weather stations located in Novosibirsk. It is expected that the air temperature at 2 meter height can differ from the surface temperature, so we were looking not for absolute equality between the time series but for a significant correlation between the two. We retrieved archived weather data for the period from 2013 to 2019 from RP5 website ${ }^{3}$. The number of usable satellite measurements at the locations of the weather stations differs from station to station and from month to month yet there are common trends and the correlation was over $90 \%$ in all cases.

We used the available satellite data to perform a qualitative assessment of the land surface temperature field and to determine patterns in the distribution of surface temperature in Novosibirsk and adjacent areas, and to identify persistent thermal anomalies. Locating such thermal anomalies may be useful for environmental management and for the evaluation of the impact of industrial zones.

Figure 1a shows the composite image based on the series of Landsat 8 images for the periods without snow (from May to September in years 2013-2019). The next Figure 1b shows the same image with land use data imposed over it. On Figure 1c the previous image

\footnotetext{
${ }^{1}$ https://developers.google.com/earth-engine/datasets/catalog/landsat

${ }^{2} \mathrm{https}: / /$ data.nextgis.com/ru/?lvl=regions\&country=ru

${ }^{3}$ https://rp5.ru
} 
is shown with the layer of roads and railways. The same process was applied to the data collected over the periods with snow (from November to March, 2013-2019)
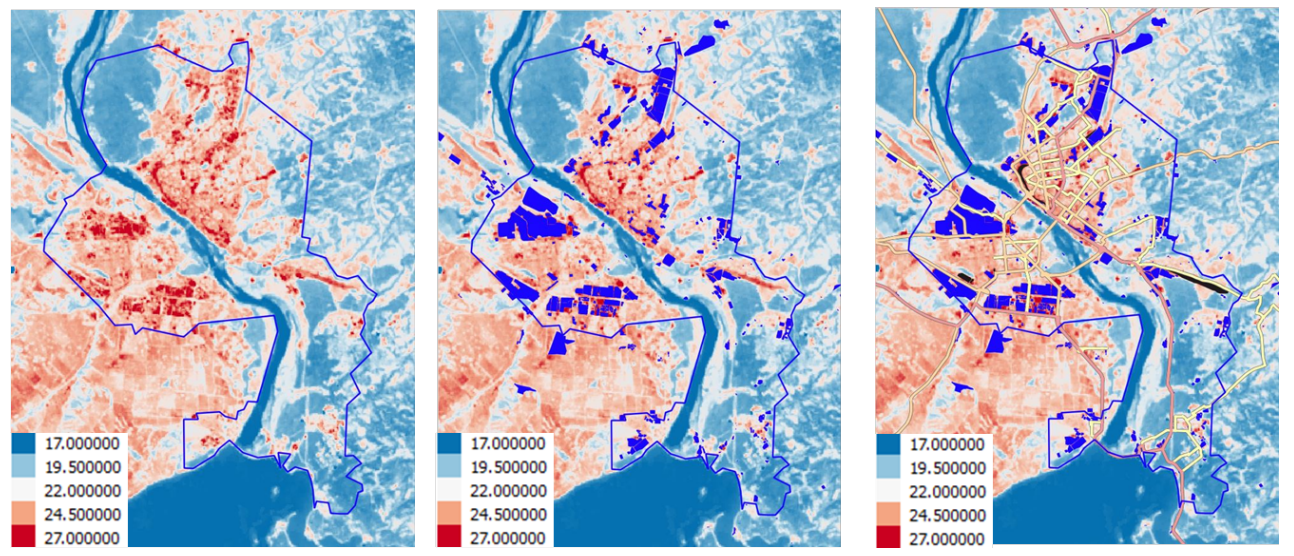

Figure 1. Temperature maps of Novosibirsk: (a) The original thermal image composite (b) composite with overlayed industrial zones (c) composite with industrial zones and road and railway networks overlayed

From Figure 1 one can see that the zones with above average surface temperature mostly correspond to industrial locations and the road network. In future we plan to perform a quantitative analysis of this link to determine the rate of growths of industrial zones.

We also studied the link between the temperature distribution and land use categories in Novosibirsk. The histogram and the temperature intervals corresponding to different categories are shown in Figure 2.
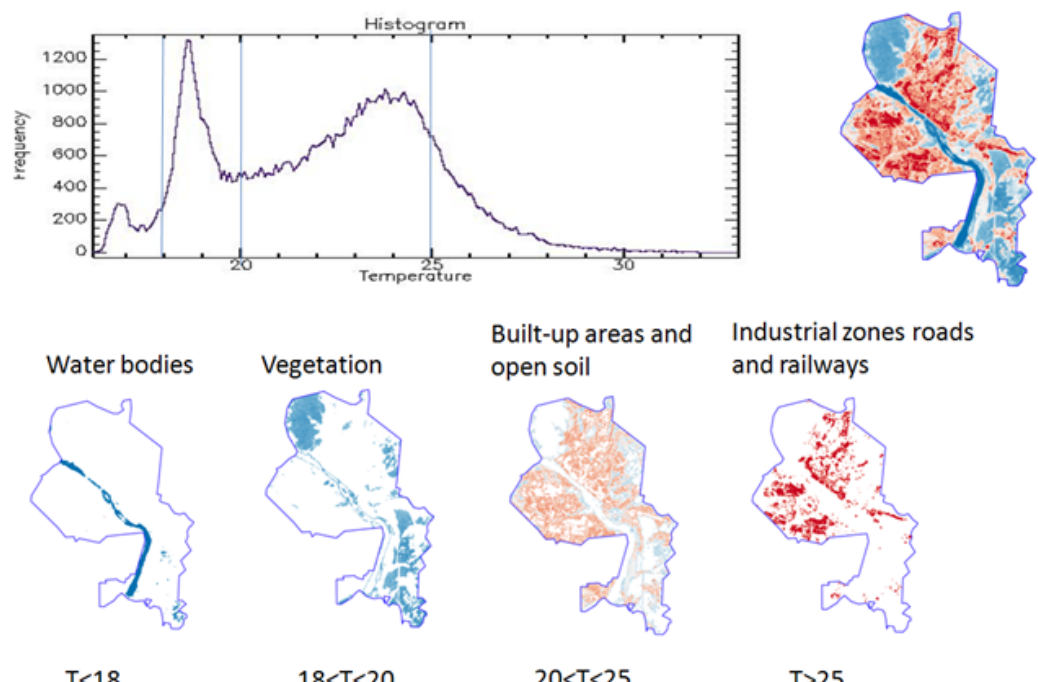

Built-up areas and

Industrial zones roads open soil and railways

Figure 2. A histogram of average temperatures in Novosibirsk and the schematic maps of the land use categories corresponding to the modes of the histogram 
Table 1. Main statistical characteristics by year

\begin{tabular}{|c|c|c|c|c|c|}
\hline Year & $\begin{array}{l}\text { Scenes } \\
\text { used }\end{array}$ & Min & Max & Mean & Stdev \\
\hline 2019 & 51 & 14.03 & 34.45 & 21.86 & 3.04 \\
\hline 2018 & 46 & 13.65 & 35.49 & 21.91 & 2.70 \\
\hline 2017 & 50 & 4.74 & 40.58 & 22.82 & 3.40 \\
\hline 2016 & 53 & 15.30 & 35.11 & 22.11 & 2.97 \\
\hline 2015 & 43 & 13.71 & 37.04 & 21.76 & 3.40 \\
\hline 2014 & 44 & 5.67 & 36.10 & 22.66 & 3.27 \\
\hline
\end{tabular}

Empirically we identified the four main land use categories within the city:

1. water bodies,

2. vegetation,

3. built-up areas and open soil,

4. industrial zones roads and railways

In future work we intend to quantify the reliability of classification into the 4 main categories based on land surface temperature. This will inform us of the practicality of using surface temperature as an additional feature for land use classification.

The temperature maps show that while the temperature distribution during the periods without snow within the boundaries of the city may change year upon year the average temperature remains relatively constant (see Figure 3, Table 1).
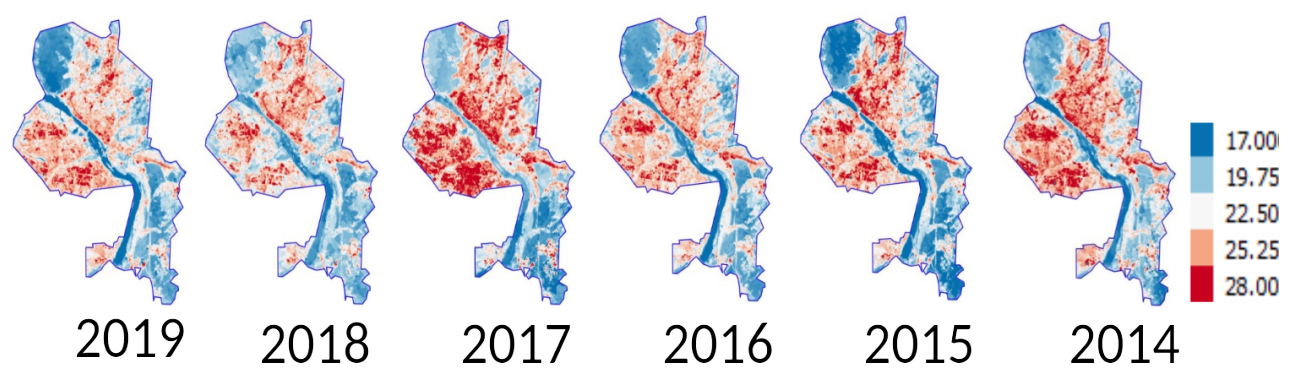

Figure 3. Spatial distribution of average temperatures during the periods without snow in Novosibirsk in 2014-2019

The results presented in this report include a method to produce land surface temperature maps based on the atmospherically corrected Landsat 8 satellite data. We confirmed that there is a strong correlation between the land surface temperature time series and air temperatures measured at weather stations at 2 meter height. Therefore land surface temperature can be used as a reliable source of information on the condition of the land cover of urban territories.

We also show that online instruments for processing of Landsat 8 time series can be used to identify spatial patterns in the land surface temperature distribution while the land surface temperature data can serve as an additional feature for classification of urban landscapes. 


\section{References}

[1] A.A. Proshin, E.A. Lupian, I.V. Balashov, A.V. Kashnitskii, and M.A. Burtsev, Current Problems in Remote Sensing of the Earth from Space, 13(3), 9-27 (2016)

[2] Yu.I. Shokin, I.A. Pestunov, D.L. Chubarov, V.A. Kikhtenko, E.A. Mamash, N.N. Dobretsov, P.V. Voronina, and Yu.N. Sinyavsky, CEUR Workshop Proceedings, 2534, 229240 (2019)

[3] E.A. Baldina and M.Y. Grishchenko, South-Eastern European Journal of Earth Observation and Geomatics, 3(2S), 415-418 (2014)

[4] M.I. Varentsov, P.I. Konstantinov, T.E. Samsonov, and I.A. Repina, Current Problems in Remote Sensing of the Earth from Space, 11(4), 329-337 (2015)

[5] M.Y. Grishchenko, M.I. Varentsov, and P. G. Mikhailyukova, Proceedings of the International Conference on Regional Problems of Remote Sensing of the Earth, 217-219 (2019)

[6] M.I. Varentsov, M.Y. Grishchenko, and H. Wouters, Geography, Environment, Sustainability, 12(4), 74-95 (2019)

[7] M.Y. Grischenko and J.V. Yermilova, Geodesy and Cartography, 79(3), 23-34 (2018)

[8] A.A. Gosteva, A.K. Matuzko and O.E. Yakubailik, Proceedings of the All-Russian Conference "Spatial Data Processing for Monitoring of Natural and Anthropogenic Processes" (SDM-2019), 387-392(2019)

[9] A.K. Matuzko, O.E. Yakubailik, Journal of Siberian Federal University, series Engineering and Technologies, 11(8), 934-945 (2018)

[10] J.C. Jiménez-Muñoz, J.A. Sobrino, D. Skoković, C. Mattar, and J. Cristóbal, IEEE Geoscience Remote Sensing Letters, 11, 1840-1843 (2014)

[11] D. Parastatidis, Z. Mitraka, N. Chrysoulakis and M. Abrams, Remote Sensing, 9(12), 1208 (2014)

[12] A. Sekertekin, and S. Bonafoni, Remote Sensing, 12(2), 294 (2020) 